\title{
Laboratory conditions in the coherent control of reactive scattering
}

\author{
Paul Brumer,* Alexander Abrashkevich and Moshe Shapiro $\dagger$ \\ Chemical Physics Theory Group, University of Toronto, and Photonics Research Ontario, \\ Toronto, Canada M5S $3 \mathrm{H} 6$
}

Received 18th March 1999

The essential requirements for preparing a bimolecular collisional superposition state in the laboratory, allowing for control over reactive scattering, are described. Specific applications to isotopic variants of $\mathrm{H}+\mathrm{H}_{2}$ scattering are used to demonstrate the range of control for superposition states built from degenerate diatomic states.

\section{Introduction}

The principle of controlling atomic and molecular processes via quantum interference, i.e. coherent control, is now well established. ${ }^{1,2}$ In particular, one arranges to arrive at a final state through two or more independent coherent routes whose features can be varied via experimentally controllable parameters. Having done so, the multiple routes, and the interference between them, can be controlled by changing the relevant laboratory parameters. Numerous computational and experimental studies show that the interference contribution is a function of the particular product so that one can alter the ratio of products by manipulating the interference contributions through laboratory variables. Further, this control is often found to be extensive.

This principle has been established formally and computationally for both unimolecular and bimolecular processes and shown experimentally for unimolecular processes. ${ }^{3-5} \mathrm{~A}$ review of this work makes clear, however, that the control of bimolecular processes is still in its infancy. In particular, we have presented the rudiments of a general formalism for control of bimolecular collisions, ${ }^{6-8}$ studied control for one-dimensional reactive scattering ${ }^{9}$ and demonstrated control of the differential cross section ${ }^{7,8}$ for isotopic variants of $\mathrm{H}+\mathrm{H}_{2}$ in a particular implementation discussed further, below. In this paper we systematically describe the specific requirements for bimolecular control and discuss methods for implementing control in the laboratory. In their most general form they invoke the manipulation of matter as waves, an area overlapping with matter interferometry ${ }^{10}$ and laser cooling. ${ }^{11}$ We then consider one simple scenario to examine the extent of possible control.

Consider then the general collision processes

$$
\mathrm{A}+\mathrm{D} \rightarrow \mathrm{F}+\mathrm{G}
$$

where A, D, F, G are, in general, molecules of mass $M_{\mathrm{A}}, M_{\mathrm{D}}, M_{\mathrm{F}}$ and $M_{\mathrm{G}}$. Here $\mathrm{F}$ and $\mathrm{G}$ can be identical to $\mathrm{A}$ and $\mathrm{D}$ (nonreactive scattering) or different from $\mathrm{A}$ and $\mathrm{D}$ (reactive scattering). We

$\dagger$ Permanent address: Department of Chemical Physics, The Weizmann Institute of Science, 76100, Rehovot, Israel. 
label $\mathrm{A}+\mathrm{D}$ as arrangement $q$ and $\mathrm{F}+\mathrm{G}$ as arrangement $q^{\prime}$. Eigenfunctions of the asymptotic reactant Hamiltonian at energy $E$, where $\mathrm{A}+\mathrm{D}$ are widely separated, are denoted $|E, q, \boldsymbol{m} ; 0\rangle$. Here $\boldsymbol{m}$ includes all quantum number which describe the state, other than the energy $E$ and the arrangement $q$. Asymptotic product states of $\mathrm{F}+\mathrm{G}$ are similarly denoted $\left|E, q^{\prime}, \boldsymbol{n} ; 0\right\rangle$, and $\mid E, q^{\prime}$, $\left.\boldsymbol{n}^{+}\right\rangle$denotes the outgoing scattering solutions ${ }^{12}$ associated with product in state $\left|E, q^{\prime}, \boldsymbol{n} ; 0\right\rangle$. The probability $P_{E}\left(\boldsymbol{n}, q^{\prime} ; \boldsymbol{m}, q\right)$ of forming the product $\left|E, q^{\prime}, \boldsymbol{n} ; 0\right\rangle$, having initiated the scattering in $\mid E$, $q, \boldsymbol{m} ; 0>$ is given by

$$
P_{E}\left(\boldsymbol{n}, q^{\prime} ; \boldsymbol{m}, q\right)=\left|\left\langle E, q^{\prime}, \boldsymbol{n} ; 0|\boldsymbol{S}| E, q, \boldsymbol{m} ; 0\right\rangle\right|^{2}
$$

where $\boldsymbol{S}$ is the scattering matrix. Alternatively, the cross section $\sigma_{E}\left(\boldsymbol{n}, q^{\prime} ; \boldsymbol{m}, q\right)$ for forming $\mid E, q^{\prime}, \boldsymbol{n}$; $0\rangle$ having initiated the scattering in $|E, q, \boldsymbol{m} ; 0\rangle$ is:

$$
\sigma_{E}\left(\boldsymbol{n}, q^{\prime} ; \boldsymbol{m}, q\right)=\left|\left\langle E, q^{\prime}, \boldsymbol{n}^{+}\left|V_{q}\right| E, q, \boldsymbol{m} ; 0\right\rangle\right|^{2} .
$$

Here $V_{q}$ is the component of the total potential that vanishes as the A to D distance becomes arbitrarily large. The cross section for scattering into arrangement $q^{\prime}$, independent of the product internal state $\boldsymbol{n}$, is

$$
\sigma_{E}\left(q^{\prime} ; \boldsymbol{m}, q\right)=\sum_{\boldsymbol{n}}\left|\left\langle E, q^{\prime}, \boldsymbol{n}^{+}\left|V_{q}\right| E, q, \boldsymbol{m} ; 0\right\rangle\right|^{2} .
$$

Assorted other cross sections may be defined, depending upon which of the elements of $\boldsymbol{n}$ are summed over. Of relevance below are (a) $\sigma_{E}\left(q^{\prime}, \theta, \phi ; \boldsymbol{m}, q\right)$, corresponding to scattering into the $q^{\prime}$ product channel and into scattering angles $(\theta, \phi)$ and (b) the traditional differential cross section $\sigma_{E}\left(q^{\prime}, \theta ; \boldsymbol{m}, q\right)=\int_{0}^{2 \pi} \mathrm{d} \phi \sigma_{E}\left(q^{\prime}, \theta, \phi ; \boldsymbol{m}, q\right)$.

Note that eqns. (2)-(4) arise in scattering theory after separating out the motion of the center of mass of A-D, a feature discussed in greater detail in Section II.

To control bimolecular collisions we construct an initial state $\left|E, q,\left\{c_{\boldsymbol{m}}\right\}\right\rangle$ consisting of a superposition of $N$ energetically degenerate asymptotic states $|E, q, \boldsymbol{m} ; 0\rangle$ :

$$
\left|E, q,\left\{c_{\boldsymbol{m}}\right\}\right\rangle=\sum_{\boldsymbol{m}} c_{\boldsymbol{m}}|E, q, \boldsymbol{m} ; 0\rangle .
$$

The cross section associated with using eqn. (5) as the initial state, obtained by replacing $\mid E, q, \boldsymbol{m}$; $0>$ by eqn. (5) in eqn. (3), is

$$
\begin{aligned}
\sigma_{E}\left(\boldsymbol{n}, q^{\prime} ;\left\{c_{\boldsymbol{m}}\right\}, q\right)= & \left|\left\langle E, q^{\prime}, \boldsymbol{n}^{+}\left|V_{q} \sum_{\boldsymbol{m}} c_{\boldsymbol{m}}\right| E, q, \boldsymbol{m} ; 0\right\rangle\right|^{2} \\
= & \left.\sum_{\boldsymbol{m}}\left|c_{\boldsymbol{m}}\right|^{2}\left\langle E, q^{\prime}, \boldsymbol{n}^{+}\left|V_{q}\right| E, q, \boldsymbol{m} ; 0\right\rangle\right|^{2} \\
& +\sum_{\boldsymbol{m}^{\prime}} \sum_{\boldsymbol{m} \neq \boldsymbol{m}^{\prime}} c_{\boldsymbol{m}} c_{\boldsymbol{m}^{\prime}}^{*}\left\langle E, q^{\prime}, \boldsymbol{n}^{+}\left|V_{q}\right| E, q, \boldsymbol{m} ; 0\right\rangle\left\langle E, q, \boldsymbol{m}^{\prime} ; 0\left|V_{q}\right| E, q^{\prime}, \boldsymbol{n}^{+}\right\rangle \\
\equiv & \sum_{\boldsymbol{m}}\left|c_{\boldsymbol{m}}\right|^{2} \sigma\left(\boldsymbol{n}, q^{\prime} ; \boldsymbol{m}, q\right)+\sum_{\boldsymbol{m}^{\prime}} \sum_{\boldsymbol{m} \neq \boldsymbol{m}^{\prime}} c_{\boldsymbol{m}} c_{\boldsymbol{m}^{\prime}}^{*} \sigma\left(\boldsymbol{n}, q^{\prime} ; \boldsymbol{m}^{\prime}, \boldsymbol{m}, q\right)
\end{aligned}
$$

where $\sigma\left(\boldsymbol{n}, q^{\prime} ; \boldsymbol{m}^{\prime}, \boldsymbol{m}, q\right)$ is defined via eqn. (6). The total cross section into arrangement $q^{\prime}$ is given by

$$
\sigma_{E}\left(q^{\prime} ;\left\{c_{\boldsymbol{m}}\right\}, q\right)=\sum_{\boldsymbol{n}} \sigma_{E}\left(\boldsymbol{n}, q^{\prime} ;\left\{c_{\boldsymbol{m}}\right\}, q\right)
$$

Note that eqn. (6), and hence eqn. (7), are now of a standard coherent control form, i.e. direct contributions from each member of the superposition, proportional to $\left|c_{m}\right|^{2}$, plus interference terms which are proportional to $c_{m} c_{m^{\prime}}^{*}$. This form has three significant features. First, it is clear that if we control the $c_{m}$, through assorted preparation methods, then we can control the interference term, and hence the scattering cross section. Second, it provides a specific functional form for control so that the experimentalist needs only determine a small number of terms (the $\sigma \mathrm{s}$ ) in order to generate the entire control map, i.e. control as a function of $c_{m}$. Third, it arises from eqn. (5), which includes only energetically degenerate terms. In particular, if eqn. (5) were to contain states of different energies $E$ and $E^{\prime}$ then the interference term would carry a factor of $\exp \left[\mathrm{i}\left(E-E^{\prime}\right) t / \hbar\right]$ which would average to zero over a period $\hbar /\left(E-E^{\prime}\right)$ and control would vanish. ${ }^{13}$ This degener- 
acy requirement, which is standard in all coherent control scenarios, proves somewhat demanding in collisional processes.

\section{Preparation of the scattering superposition}

To see how the required superposition state [eqn. (5)] can be constructed in the laboratory requires some introductory remarks. Note first that eqns. (2)-(7) and the $|E, q, \boldsymbol{m} ; 0\rangle$ are expressed in the center of mass coordinate system and describe the relative translational as well as the internal state of $\mathrm{A}$ and $\mathrm{D}$. In typical A-D scattering the removal of the center of mass motion comes about in a straightforward way. That is, let $\boldsymbol{r}_{\mathrm{A}}$ and $\boldsymbol{r}_{\mathrm{D}}$ denote the laboratory position of A and D and $\hbar \boldsymbol{k}^{\mathrm{A}}, \hbar \boldsymbol{k}^{\mathrm{D}}$ denote their laboratory momenta. The relative momentum $\boldsymbol{k}$, relative coordinate $\boldsymbol{r}$, center of mass momentum $\boldsymbol{K}$ and position $\boldsymbol{R}$ are defined as

$$
\begin{aligned}
\boldsymbol{K} & =\boldsymbol{k}^{\mathrm{A}}+\boldsymbol{k}^{\mathrm{D}} ; \quad \boldsymbol{R}=\left(M_{\mathrm{A}} \boldsymbol{r}_{\mathrm{A}}+M_{\mathrm{D}} \boldsymbol{r}_{\mathrm{D}}\right) /\left(M_{\mathrm{A}}+M_{\mathrm{D}}\right) \\
\boldsymbol{k} & =\left(M_{\mathrm{D}} \boldsymbol{k}^{\mathrm{A}}-M_{\mathrm{A}} \boldsymbol{k}^{\mathrm{D}}\right) /\left(M_{\mathrm{A}}+M_{\mathrm{D}}\right) ; \quad \boldsymbol{r}=\boldsymbol{r}_{\mathrm{A}}-\boldsymbol{r}_{\mathrm{D}} .
\end{aligned}
$$

In the case where $\mathrm{A}$ and $\mathrm{D}$ are initially in internal states $\left|\phi_{\mathrm{A}}(i)\right\rangle$ and $\left|\phi_{\mathrm{D}}(j)\right\rangle$, of energies $E_{\mathrm{A}}(i)$ and $E_{\mathrm{D}}(j)$, and the initial A and D translational motions are described by plane waves of momenta $\boldsymbol{k}_{i}^{\mathrm{A}}$ and $\boldsymbol{k}_{j}^{\mathrm{D}}$ then the incident wavefunction $\psi_{\text {in }}$ is

$$
\begin{aligned}
\psi_{\text {in }} & =\left|\phi_{\mathrm{A}}(i)\right\rangle\left|\phi_{\mathrm{D}}(j)\right\rangle \exp \left(\mathrm{i} \boldsymbol{k}_{i}^{\mathrm{A}} \cdot \boldsymbol{r}_{\mathrm{A}}\right) \exp \left(\mathrm{i} \boldsymbol{k}_{j}^{\mathrm{D}} \cdot \boldsymbol{r}_{\mathrm{D}}\right) \\
& =\left|\phi_{\mathrm{A}}(i)\right\rangle\left|\phi_{\mathrm{D}}(j)\right\rangle \exp (\mathrm{i} \boldsymbol{k} \cdot \boldsymbol{r}) \exp (\mathrm{i} \boldsymbol{K} \cdot \boldsymbol{R}) .
\end{aligned}
$$

The second equality follows from eqns. (8). Since the interaction potential $V_{q}$ between A and D depends solely upon the A-D relative coordinates, the center of mass momentum is conserved in the collision, allowing us to separate out the center of mass motion and to describe the dynamics in the center of mass coordinate system, i.e., in terms of $|E, q, \boldsymbol{m} ; 0\rangle$.

Scattering may also occur from a state composed of different values of center of mass momenta $\boldsymbol{K}$. For example,

$$
\left|\psi_{\text {in }}\right\rangle=\sum_{l \boldsymbol{m}} d_{l \boldsymbol{m}}|E, q, \boldsymbol{m} ; 0\rangle\left|\boldsymbol{K}_{l}\right\rangle \quad\left(\boldsymbol{K}_{l^{\prime}} \neq \boldsymbol{K}_{l}\right)
$$

Since the center of mass momentum is conserved, and since it can be measured at the end of the collision, the cross section for scattering into $\left|E, q^{\prime}, \boldsymbol{n} ; 0\right\rangle$ in this case is given by

$$
\sigma_{E}\left(\boldsymbol{n}, q^{\prime} ;\left\{d_{l \boldsymbol{m}}\right\}, q\right)=\sum_{l}\left|\left\langle E, q^{\prime}, \boldsymbol{n}^{+}\left|V_{q} \sum_{\boldsymbol{m}} d_{l \boldsymbol{m}}\right| E, q, \boldsymbol{m} ; 0\right\rangle\right|^{2} .
$$

That is, components of the wavefunction with different values of $\left|\boldsymbol{K}_{l}\right\rangle$ contribute independently to the reaction cross section and do not interfere with one another.

Consider now preparation of the generalized superposition states [eqn. (5)] where, for simplicity, we limit consideration to the preparation of a superposition of two states. To do so we examine the scattering of $\mathrm{A}$ and $\mathrm{D}$, each in a previously prepared superposition state. The wavefunctions $\psi_{\mathrm{A}}$ and $\psi_{\mathrm{D}}$ in the laboratory system are chosen to be of the general form:

$$
\begin{aligned}
& \psi_{\mathrm{A}}=a_{1}\left|\phi_{\mathrm{A}}(1)\right\rangle \exp \left(\mathrm{i} \boldsymbol{k}_{1}^{\mathrm{A}} \cdot \boldsymbol{r}_{\mathrm{A}}\right)+a_{2}\left|\phi_{\mathrm{A}}(2)\right\rangle \exp \left(\mathrm{i} \boldsymbol{k}_{2}^{\mathrm{A}} \cdot \boldsymbol{r}_{\mathrm{A}}\right) \\
& \psi_{\mathrm{D}}=a_{3}\left|\phi_{\mathrm{D}}(3)\right\rangle \exp \left(\mathrm{i}_{3}^{\mathrm{D}} \cdot \boldsymbol{r}_{\mathrm{D}}\right)+a_{4}\left|\phi_{\mathrm{D}}(4)\right\rangle \exp \left(\boldsymbol{i}_{4}^{\mathrm{D}} \cdot \boldsymbol{r}_{\mathrm{D}}\right) .
\end{aligned}
$$

Since $\mathrm{A}$ and $\mathrm{D}$ are initially noninteracting, the incident wavefunction is

$$
\begin{aligned}
\psi_{\mathrm{in}}= & \psi_{\mathrm{A}} \psi_{\mathrm{D}}=\left[a_{1}\left|\phi_{\mathrm{A}}(1)\right\rangle \exp \left(\mathrm{i} \boldsymbol{k}_{1}^{\mathrm{A}} \cdot \boldsymbol{r}_{\mathrm{A}}\right)+a_{2}\left|\phi_{\mathrm{A}}(2)\right\rangle \exp \left(\mathrm{i} \boldsymbol{k}_{2}^{\mathrm{A}} \cdot \boldsymbol{r}_{\mathrm{A}}\right)\right] \\
& \times\left[a_{3}\left|\phi_{\mathrm{D}}(3)\right\rangle \exp \left(\mathrm{i} \boldsymbol{k}_{3}^{\mathrm{D}} \cdot \boldsymbol{r}_{\mathrm{D}}\right)+a_{4}\left|\phi_{\mathrm{D}}(4)\right\rangle \exp \left(\mathrm{i}_{4}^{\mathrm{D}} \cdot \boldsymbol{r}_{\mathrm{D}}\right)\right] \\
= & \sum_{i=1}^{2} \sum_{j=3}^{4} A_{i j} \exp \left(\mathrm{i}_{i j} \cdot \boldsymbol{r}\right) \exp \left(\mathrm{i} \boldsymbol{K}_{i j} \cdot \boldsymbol{R}\right)
\end{aligned}
$$

where $A_{i j}=a_{i} a_{j}\left|\phi_{\mathrm{A}}(i)\right\rangle\left|\phi_{\mathrm{D}}(j)\right\rangle, \quad \boldsymbol{k}_{i j}=\left(M_{\mathrm{D}} \boldsymbol{k}_{i}^{\mathrm{A}}-M_{\mathrm{A}} \boldsymbol{k}_{j}^{\mathrm{D}}\right) /\left(M_{\mathrm{A}}+M_{\mathrm{D}}\right), \quad$ and $\boldsymbol{K}_{i j}=\boldsymbol{k}_{i}^{\mathbf{A}}+\boldsymbol{k}_{j}^{\mathrm{D}}$ with $(i=1,2 ; j=3,4)$. 
As constructed, eqn. (14) is composed of four independent noninterfering incident wavepackets since each has a different center of mass wavevector $\boldsymbol{K}_{i j}$. However, we can set conditions so that interference, and hence control, is indeed achieved. That is, we require the equality of the center of mass motion of two components, plus energy degeneracy:

$$
\begin{aligned}
\boldsymbol{K}_{13} & =\boldsymbol{K}_{24} \\
\hbar^{2} k_{13}^{2} / 2 \mu+e_{\mathrm{A}}(1)+e_{\mathrm{D}}(3) & =\hbar^{2} k_{24}^{2} / 2 \mu+e_{\mathrm{A}}(2)+e_{\mathrm{D}}(4),
\end{aligned}
$$

with $\mu=M_{\mathrm{A}} M_{\mathrm{D}} /\left(M_{\mathrm{A}}+M_{\mathrm{D}}\right)$. Eqn. (14) then becomes

$$
\begin{aligned}
\psi_{\mathrm{in}}= & {\left[A_{13} \exp \left(\mathrm{i}_{13} \cdot \boldsymbol{r}\right)+A_{24} \exp \left(\mathrm{i}_{24} \cdot \boldsymbol{r}\right)\right] \exp \left(\mathrm{i}_{13} \cdot \boldsymbol{R}\right) } \\
& +A_{23} \exp \left(\mathrm{i}_{23} \cdot \boldsymbol{r}\right) \exp \left(\mathrm{i}_{23} \cdot \boldsymbol{R}\right)+A_{14} \exp \left(\mathrm{i} \boldsymbol{k}_{14} \cdot \boldsymbol{r}\right) \exp \left(\boldsymbol{i}_{14} \cdot \boldsymbol{R}\right),
\end{aligned}
$$

where the term in the first bracket, due to eqn. (15), is a linear superposition of two degenerate states. In accord with eqns. (10) and (11) we expect that the scattering cross section will be composed of noninterfering contributions from three components with different $\boldsymbol{K}_{i j}$, but where the first term allows for control via the interference between the $A_{13}$ and $A_{24}$ terms. The two remaining terms, proportional to $A_{23}$ and $A_{14}$, are uncontrolled satellite contributions.

For example, if we set $\boldsymbol{k}_{1}^{\mathrm{A}}=-\boldsymbol{k}_{3}^{\mathrm{D}}$ and $\boldsymbol{k}_{2}^{\mathrm{A}}=-\boldsymbol{k}_{4}^{\mathrm{D}}$ then $\boldsymbol{K}_{13}=\boldsymbol{K}_{24}=0$. In addition, $\boldsymbol{k}_{13}=\boldsymbol{k}_{1}^{\mathrm{A}}$, $\boldsymbol{k}_{24}=\boldsymbol{k}_{2}^{\mathrm{A}}$ so that eqn. (15) becomes

$$
\hbar^{2}\left[\left(k_{1}^{\mathrm{A}}\right)^{2}-\left(k_{2}^{\mathrm{A}}\right)^{2}\right]=\left(2 M_{\mathrm{D}} M_{\mathrm{A}}\right)\left[e_{\mathrm{A}}(2)+e_{\mathrm{D}}(4)-e_{\mathrm{A}}(1)-e_{\mathrm{D}}(3)\right] /\left(M_{\mathrm{D}}-M_{\mathrm{A}}\right)
$$

Note also that we can implement eqn. (17) for the case of atom-diatom scattering by setting $\left|\phi_{\mathrm{A}}(1)\right\rangle=\left|\phi_{\mathrm{A}}(2)\right\rangle=\left|\phi_{\mathrm{A}}(\mathrm{g})\right\rangle$, where $\left|\phi_{\mathrm{A}}(\mathrm{g})\right\rangle$ is the ground electronic state of atom A. Eqn. (17) then becomes

$$
\hbar^{2}\left[\left(k_{1}^{\mathrm{A}}\right)^{2}-\left(k_{2}^{\mathrm{A}}\right)^{2}\right]=\left(2 M_{\mathrm{D}} M_{\mathrm{A}}\right)\left[e_{\mathrm{D}}(4)-e_{\mathrm{D}}(3)\right] /\left(M_{\mathrm{D}}-M_{\mathrm{A}}\right)
$$

Finally, in the specific case where we superpose degenerate states of the molecule D, eqn. (18) becomes $\left(k_{1}^{\mathbf{A}}\right)^{2}=\left(k_{2}^{\mathbf{A}}\right)^{2}$. Indeed, in this case we can choose $\boldsymbol{k}_{1}^{\mathbf{A}}=\boldsymbol{k}_{2}^{\mathbf{A}}=-\boldsymbol{k}_{3}^{\mathbf{D}}=-\boldsymbol{k}_{4}^{\mathrm{D}}$ so that all of the $\boldsymbol{K}_{i j}$ are equal and all of the four terms in eqn. (14) are energetically degenerate in the center of mass coordinate system. Hence in this case, which corresponds to the scattering of an atom off a molecule in a superposition of degenerate molecular states, there are no extraneous uncontrollable satellite contributions. Results for this case are considered below for the case of atom-diatom scattering.

\section{M Superpositions}

It is clear that the easiest way of implementing bimolecular control in the laboratory is to start with a superposition of degenerate states of the fragments. In atom-diatom scattering, to which we restrict attention, we utilize the $(2 j+1)$ diatomic rotational states $|j, m\rangle$, where $m$ is the projection of diatomic angular momentum $j$ along a space fixed axis. In this case (and in the case of the helicity, i.e. the projection of the angular momentum along the relative velocity vector, ${ }^{7,8}$ as well) we show below that control over the differential cross section is possible but control over the total cross section is not.

Consider first superimposing two $m$ states of the diatomic in atom-diatom scattering. For this case eqn. (5) assumes the form:

$$
|\boldsymbol{n}, q\rangle=\sum_{i=1,2} c_{i}\left|q v j m_{i}\right\rangle\left|E_{q}^{\mathrm{kin}}\right\rangle,
$$

where $\left|q v j m_{i}\right\rangle$ is an eigenket of the diatomic of energy $\epsilon_{q v j}$, with $v$ denoting the diatom vibrational quantum number. $\left|E_{q}^{\text {kin }}\right\rangle$ is a plane wave of energy $E_{q}^{\text {kin }}=E-\epsilon_{q v j}$, describing the free motion of the atom relative to the diatom in the $q$ arrangement.

The differential cross section into scattering angles $\theta, \varphi$ is given by

$$
\sigma\left(q^{\prime} v^{\prime} j^{\prime} k^{\prime} \leftarrow q, v, j, m_{1}, m_{2} \mid \theta, \varphi\right)=\left|\sum_{i=1,2} c_{i} f_{q^{\prime} v^{\prime} j^{\prime} k^{\prime} \leftarrow q v j m_{i}}(\pi-\theta, \varphi)\right|^{2} .
$$


Here

$$
f_{q^{\prime} v^{\prime} j^{\prime} k^{\prime} \leftarrow q v j m_{i}}(\theta, \varphi)=\frac{i^{j-j^{\prime}+1} \mathrm{e}^{\mathrm{i} m_{i} \varphi}}{2 k_{q v j}} \sum_{J}(2 J+1) d_{k^{\prime} m_{i}}^{J}(\theta) T_{q^{\prime} v^{\prime} j^{\prime} k^{\prime}, q v j m_{i}}
$$

is the scattering amplitude, ${ }^{14} T_{q^{\prime} v^{\prime} j^{\prime} k^{\prime}, q v j m_{i}}^{J}=S_{q^{\prime} v^{\prime} j^{\prime} k^{\prime}, q v j m_{i}}^{J}-\delta_{q^{\prime} q} \delta_{v^{\prime} v} \delta_{j^{\prime} j} \delta_{k^{\prime} m_{i}}$, and $S_{q^{\prime} v^{\prime} j^{\prime} k^{\prime}, q v j m_{i}}^{J}$ are the elements of the scattering $S$-matrix in the helicity representation. ${ }^{15}$ Here $k^{\prime}$ is the helicity of the product diatom (i.e. the projection of the product diatom angular momentum onto the final relative translational velocity vector), and $k_{q v j}=\sqrt{2 \mu_{q}\left(E-\epsilon_{q v j}\right)} / \hbar$, with $\mu_{q}$ being the atom-diatom reduced mass in the $q$ channel. Note that the use of $(\pi-\theta)$ in eqn. (20), rather than $\theta$, is discussed in ref. 16.

Expanding the square in eqn. (20) gives the reactive differential scattering cross section as (where we drop the initial state labels for convenience)

$$
\sigma^{\mathrm{R}}\left(v^{\prime} j^{\prime} k^{\prime} \mid \theta, \varphi\right)=\left|c_{1}\right|^{2} \sigma_{11}^{\mathrm{R}}\left(v^{\prime} j^{\prime} k^{\prime} \mid \theta\right)+\left|c_{2}\right|^{2} \sigma_{22}^{\mathrm{R}}\left(v^{\prime} j^{\prime} k^{\prime} \mid \theta\right)+2 \operatorname{Re}\left\{c_{1}^{*} c_{2} \sigma_{12}^{\mathrm{R}}\left(v^{\prime} j^{\prime} k^{\prime} \mid \theta, \varphi\right)\right\},
$$

where

$$
\begin{aligned}
\sigma_{i i}^{\mathrm{R}}\left(v^{\prime} j^{\prime} k^{\prime} \mid \theta\right)= & \left|f_{q^{\prime} v^{\prime} j^{\prime} k^{\prime} \leftarrow q v j m_{i}}(\pi-\theta, \varphi)\right|^{2} \\
= & \left(4 k_{q v j}^{2}\right)^{-1} \sum_{J, J^{\prime}}(2 J+1)\left(2 J^{\prime}+1\right) d_{k^{\prime} m_{i}}^{J}(\pi-\theta) d_{k^{\prime} m_{i}}^{J^{\prime}}(\pi-\theta) \\
& \times S_{q^{\prime} v^{\prime} j^{\prime} k^{\prime}, q v j m_{i}}^{J}\left[S_{q^{\prime} v^{\prime} j^{\prime} k^{\prime}, q v j m_{i}}^{J^{\prime}}\right]^{*}, \quad q \neq q^{\prime}, \quad i=1,2,
\end{aligned}
$$

and

$$
\begin{aligned}
\sigma_{12}^{\mathrm{R}}\left(v^{\prime} j^{\prime} k^{\prime} \mid \theta, \varphi\right)= & f_{q^{\prime} v^{\prime} j^{\prime} k^{\prime} \leftarrow q v j m_{i}}(\pi-\theta, \varphi) f_{q^{\prime} v^{\prime} j^{\prime} k^{\prime} \leftarrow q v j m_{i}}^{*}(\pi-\theta, \varphi) \\
= & \frac{\mathrm{e}^{\mathrm{i}\left(m_{1}-m_{2}\right) \varphi}}{4 k_{q v j}^{2}} \sum_{J, J^{\prime}}(2 J+1)\left(2 J^{\prime}+1\right) \\
& \times d_{k^{\prime} m_{1}}^{J}(\pi-\theta) d_{k^{\prime} m_{2}}^{J^{\prime}}(\pi-\theta) S_{q^{\prime} v^{\prime} j^{\prime} k^{\prime}, q v j m_{1}}^{J}\left[S_{q^{\prime} v^{\prime} j^{\prime} k^{\prime}, q v j m_{2}}^{J^{\prime}}\right]^{*}, \quad q \neq q^{\prime} .
\end{aligned}
$$

Here, the superscript $\mathbf{R}$ denotes reactive scattering into a specific final arrangement channel $q^{\prime} \neq q$. The total differential cross section, $\sigma^{\mathrm{R}}(\theta, \varphi)$, for reaction out of a state in eqn. (19) is given by the sum over final states at energy $E$ as

$$
\sigma^{\mathrm{R}}(\theta, \varphi)=\sum_{v^{\prime}, j^{\prime}, k^{\prime}} \sigma^{\mathrm{R}}\left(v^{\prime} j^{\prime} k^{\prime} \mid \theta, \varphi\right)
$$

Note that the $\varphi$ dependence of the measurable cross sections is due solely to the interference term. Integration of eqn. (22) or (25) over angles $\theta \in[0, \pi]$ and $\varphi \in[0,2 \pi]$ gives the state-resolved integral reactive cross section $\sigma^{\mathrm{R}}\left(v^{\prime} j^{\prime} k^{\prime}\right)$. However, the integral of $\sigma_{12}^{\mathrm{R}}\left(v^{\prime} j^{\prime} k^{\prime} \mid \theta, \varphi\right)$ over $\varphi$ is zero so that the interference term, and hence control over the total cross section disappears. Indeed, the integral over $0<\varphi<\pi$ exactly cancels the integral over $\pi<\varphi<2 \pi$. For this reason we consider control over scattering into the hemisphere $0<\varphi<\pi$, giving the state resolved integral cross section denoted $\sigma^{\mathrm{R}}\left(v^{\prime} j^{\prime} k^{\prime} ; \varphi \leqslant \pi\right)$. This can also be written as three terms, as in eqn. (22), but with the $\sigma_{i j}^{\mathrm{R}}$ replaced by $\sigma_{i j}^{\mathrm{R}}\left(v^{\prime} j^{\prime} k^{\prime} ; \varphi \leqslant \pi\right),(i, j=1,2)$, where

$$
\begin{aligned}
& \sigma_{i i}^{\mathrm{R}}\left(v^{\prime} j^{\prime} k^{\prime} ; \varphi \leqslant \pi\right)=\frac{\pi}{2 k_{q v j}^{2}} \sum_{J}(2 J+1)\left|S_{q^{\prime} v^{\prime} j^{\prime} k^{\prime}, q v j m_{i}}^{J}\right|^{2}, \quad i=1,2, \\
& \sigma_{12}^{\mathrm{R}}\left(v^{\prime} j^{\prime} k^{\prime} ; \varphi \leqslant \pi\right)=\left(4 k_{q v j}^{2}\right)^{-1} \sum_{J, J^{\prime}}(2 J+1)\left(2 J^{\prime}+1\right) S_{q^{\prime} v^{\prime} j^{\prime} k^{\prime}, q v j m_{1}}^{J}\left[S_{q^{\prime} v^{\prime} j^{\prime} k^{\prime}, q v j m_{2}}^{J^{\prime}}\right]^{*} \\
& \times I\left(m_{1}, m_{2}\right) \int_{0}^{\pi} \mathrm{d} \theta \sin \theta d_{k^{\prime} m_{1}}^{J}(\pi-\theta) d_{k^{\prime} m_{2}}^{J^{\prime}}(\pi-\theta), \quad q \neq q^{\prime},
\end{aligned}
$$

with

$$
I\left(m_{1}, m_{2}\right)=\int_{0}^{\pi} \mathrm{d} \varphi \mathrm{e}^{\mathrm{i}\left(m_{1}-m_{2}\right) \varphi}= \begin{cases}\frac{2 \mathrm{i}}{m_{1}-m_{2}}, & \text { if } m_{1}-m_{2} \text { is odd } \\ 0, & \text { if } m_{1}-m_{2} \text { is even } .\end{cases}
$$


Summing over the final $v^{\prime}, j^{\prime}, k^{\prime}$ at energy $E$, gives the total integral cross section into the hemisphere as

$$
\sigma^{\mathrm{R}}(\varphi \leqslant \pi)=\sum_{v^{\prime}, j^{\prime}, k^{\prime} ; \varphi \leqslant \pi} \sigma^{\mathrm{R}}\left(v^{\prime}, j^{\prime}, k^{\prime}\right)
$$

It is important to stress that state-to-state cross sections $\sigma_{i i}^{\mathrm{R}}\left(v^{\prime} j^{\prime} k^{\prime} \mid \theta\right)$ and $\sigma_{i i}^{\mathrm{R}}\left(v^{\prime} j^{\prime} k^{\prime} ; \varphi \leqslant \pi\right)$ in eqns. (23) and (26), as well as the corresponding total cross sections $\sigma_{i i}^{\mathrm{R}}(\theta)$ and $\sigma_{i i}^{\mathrm{R}}$, appear in standard scattering theory (see, e.g., ref. 17), while $\sigma_{12}^{\mathrm{R}}\left(v^{\prime} j^{\prime} k^{\prime} \mid \theta, \varphi\right)$ and $\sigma_{12}^{\mathrm{R}}\left(v^{\prime} j^{\prime} k^{\prime} ; \varphi \leqslant \pi\right)$ in eqns. (24) and (27) are new types of interference terms which allow for control, through the $c_{i}$, over the atom-diatom collision process. Note that significant control requires substantial $\sigma_{12}^{\mathrm{R}}$ which follows from the Schwartz inequality $\left[\left|\sigma_{12}^{\mathrm{R}}\right| \leqslant \sqrt{\sigma_{11}^{\mathrm{R}} \sigma_{22}^{\mathrm{R}}}\right]$, i.e. large $\sigma_{12}^{\mathrm{R}}$ requires also large $\sigma_{11}^{\mathrm{R}}$ and $\sigma_{22}^{\mathrm{R}}$. Therefore, extensive control is not limited to regions near the reactive threshold. ${ }^{18}$

To examine the extent of control over the reaction it is convenient to rewrite the reactive cross section in the form (where we refer to scattering into a hemisphere, but drop the notation " $\varphi \leqslant \pi$ " for convenience),

$$
\sigma^{\mathrm{R}}=\left[\sigma_{11}^{\mathrm{R}}+x^{2} \sigma_{22}^{\mathrm{R}}+2 x\left|\sigma_{12}^{\mathrm{R}}\right| \cos \left(\delta_{12}^{\mathrm{R}}+\phi_{12}\right)\right] /\left(1+x^{2}\right),
$$

where $x=\left|c_{2} / c_{1}\right|, \phi_{12}=\arg \left(c_{2} / c_{1}\right)$, and $\delta_{12}^{\mathrm{R}}=\arg \left(\sigma_{12}^{\mathrm{R}}\right)$. In many cases, of greatest interest is control over branching ratio between the reactive and nonreactive total cross sections which is given by

$$
\frac{\sigma^{\mathrm{R}}}{\sigma^{\mathrm{NR}}}=\frac{\sigma_{11}^{\mathrm{R}}+x^{2} \sigma_{22}^{\mathrm{R}}+2 x\left|\sigma_{12}^{\mathrm{R}}\right| \cos \left(\delta_{12}^{\mathrm{R}}+\phi_{12}\right)}{\sigma_{11}^{\mathrm{NR}}+x^{2} \sigma_{22}^{\mathrm{NR}}+2 x\left|\sigma_{12}^{\mathrm{NR}}\right| \cos \left(\delta_{12}^{\mathrm{NR}}+\phi_{12}\right)} .
$$

Here NR refers to nonreactive scattering; definitions of the nonreactive cross sections are analogous to their reactive counterparts.

It follows from eqns. (29) and (30) that by varying the $c_{i}$ coefficients (i.e. varying either the relative magnitude, $x$ or the relative phase, $\phi_{12}$ ) in eqn. (19) through the initial preparation step, we can directly alter the interference term $\sigma_{12}^{\mathrm{R}}\left(\right.$ and/or $\left.\sigma_{12}^{\mathrm{NR}}\right)$ and hence control the scattering cross sections. Such a preparation might be carried out, for example, by a suitably devised molecular beam experiment where the diatomic is excited, with elliptically polarized light to a collection of well-defined $m$ states.

Note that the above approach can be readily extended to the case of $N$ interfering superposition states, as discussed in Section IIIB below.

\section{A. Computational results}

We have considered control over the reactions $\mathrm{D}+\mathrm{H}_{2}, \mathrm{H}+\mathrm{D}_{2}$, and $\mathrm{H}+\mathrm{H}^{\prime} \mathrm{D}\left(\mathrm{H}\right.$ and $\mathrm{H}^{\prime}$ denote hydrogen atoms which are deemed distinguishable) using this approach and present a few typical results in this section. Control over reaction products is achieved by preparing superpositions of two initial degenerate interfering diatomic states $\left(v, j, m_{1}\right)$ and $\left(v, j, m_{2}\right)$. In our three-dimensional quantum-mechanical calculations, the $\mathrm{A}+\mathrm{B}_{2}$ symmetry has been exploited for the first two reactions, while the last one has been considered as an atom-heteronuclear diatom reaction of the general $\mathrm{A}+\mathrm{BC}$ type. The cross sections reported below are obtained via the symmetrization procedure described in ref. 16. Since only initial states of $\mathrm{H}_{2}$ and $\mathrm{D}_{2}$ with even $j$ were considered, only the "plus" molecular parity block was computed for each partial wave. The LSTH ${ }^{19}$ potential surface was used and scattering calculations were done with the log-derivative version of the Kohn variational principle ${ }^{20}$ using a basis set contraction procedure. ${ }^{21}$ Scattering calculations were carried out for total angular momentum up to $J=31$ with $j_{\max }=14$ and $E_{\max }=2.5 \mathrm{eV}$ for the $\mathrm{D}+\mathrm{H}_{2}$ and $\mathrm{H}+\mathrm{D}_{2}$ reactions, and up to $J=46, j_{\max }=15$ and $E_{\max }=2.6 \mathrm{eV}$ for the $\mathrm{H}+\mathrm{H}^{\prime} \mathrm{D}$ reaction, ensuring fully converged cross sections for the chosen energies. Here, $E_{\max }$ and $j_{\max }$ are, respectively, the maximum (cutoff) energy and maximum diatomic rotational quantum number of all asymptotic channels and internal basis functions included in the calculation.

Consider, as an example, the results for scattering from a superposition state consisting of the $\left(v=1, j=2, m_{1}=1, m_{2}=0\right)$, as shown in Fig. 1. Results are reported as contour plots of cross section ratios $v s$. the phase $\phi_{12}$ and the parameter $s=x^{2} /\left(1+x^{2}\right)$. The value $s=0$ corresponds to scattering from the state with $m_{1}=1$ and $s=1$ corresponds to scattering from the state with 


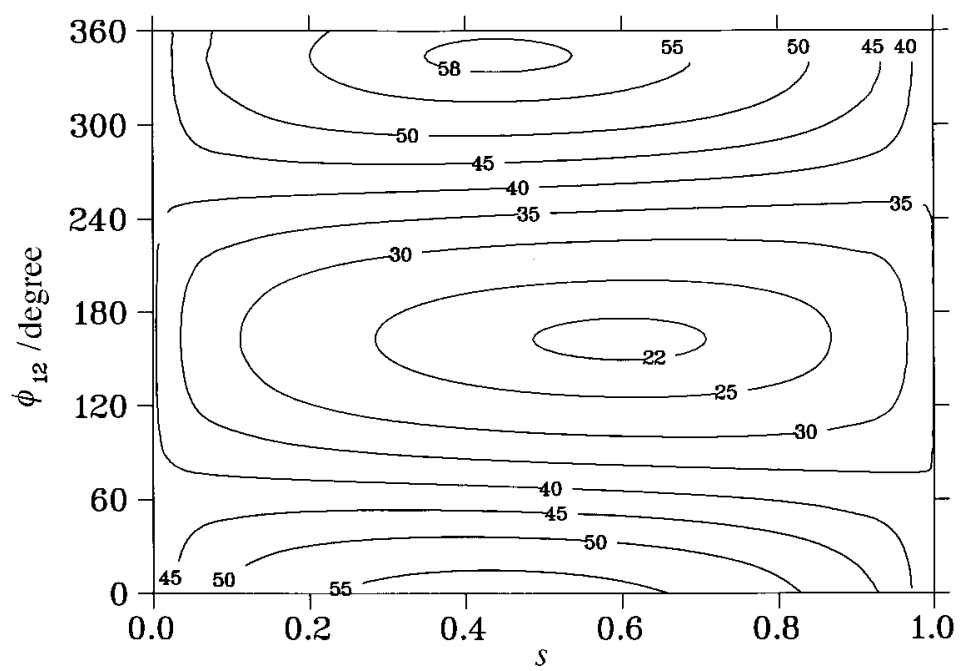

Fig. 1 Contour plot of the $\sigma^{\mathrm{R}}(\theta ; \varphi \leqslant \pi) / \sigma^{\mathrm{NR}}(\theta ; \varphi \leqslant \pi)\left(\times 10^{2}\right)$ ratio for $\mathrm{D}+\mathrm{H}_{2}$ as a function of $\phi_{12}$ and $s$ at $\theta=60^{\circ}$, with $E=1.25 \mathrm{eV}$. The initial state is a superposition state with $v=1, j=2, m_{1}=1, m_{2}=0$.

$m_{2}=0$. Fig. 1 shows $\sigma^{\mathrm{R}}(\theta ; \varphi \leqslant \pi) / \sigma^{\mathrm{NR}}(\varphi \leqslant \pi)(\theta)$, which is seen to vary from 0.22 to 0.58 as $\phi_{12}$ is changed from $157^{\circ}$ to $342^{\circ}$ at $s \approx 0.51$. This range should be compared with the uncontrolled ratios of $\sim 0.36$ at $s=1$ and $\sim 0.40$ at $s=0$. Note further that Fig. 1 is of the familiar form, ${ }^{1,2}$ resulting from the interference between two states. Thus, varying the coefficients $c_{i}$ allows one to either increase or decrease the ratio of differential cross sections, in comparison to the uncontrolled ratio, by a factor of $\sim 1.5$. Less extensive control was observed, for example, for scattering from the $\left(v=0, j=2, m_{1}=2, m_{2}=1\right)$ initial state (not shown).

Similar ranges of control are observed for control over the cross section into the hemisphere, i.e. $\sigma^{\mathrm{R}}(\varphi \leqslant \pi) / \sigma^{\mathrm{NR}}(\varphi \leqslant \pi)$, shown in Figs. 2 and 3. The results shown in Fig. 2 correspond to $\mathrm{D}+\mathrm{H}_{2} \rightarrow \mathrm{H}+\mathrm{HD}$ at $E=0.93 \mathrm{eV}$, with scattering from an initial superposition of $(v=0, j=2$, $m_{1}=1, m_{2}=0$ ). The ratio of cross sections is seen to vary from 0.05 to 0.079 , showing maximum and minimum that are well outside (up to factor 1.22 and 1.26 respectively) of the range of results

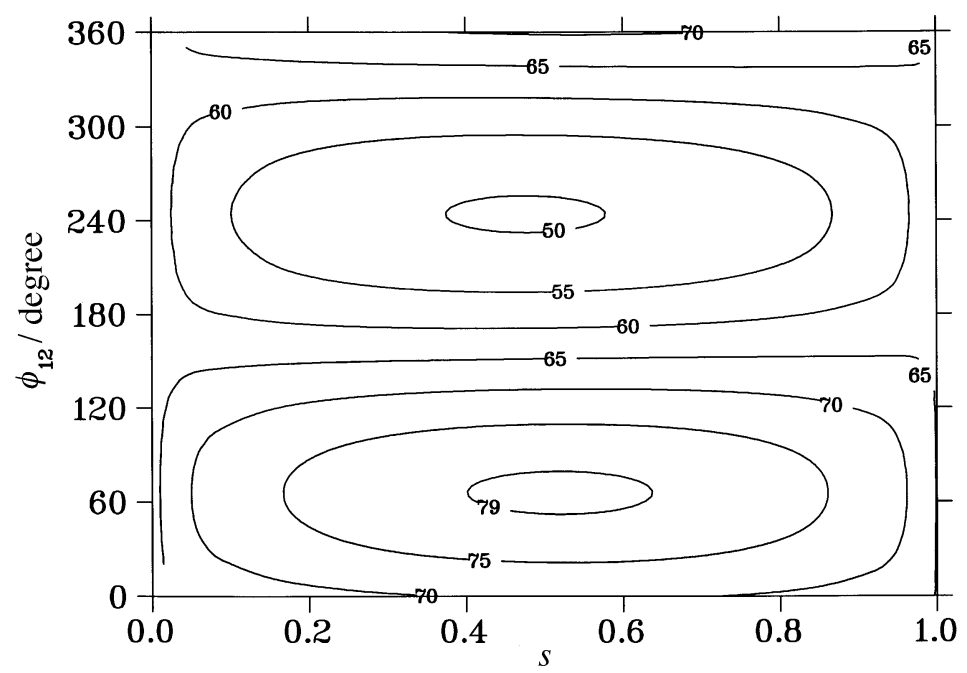

Fig. 2 Contour plot of the ratio $\sigma^{\mathrm{R}}(\varphi \leqslant \pi) / \sigma^{\mathrm{NR}}(\varphi \leqslant \pi)\left(\times 10^{3}\right)$ for $\mathrm{D}+\mathrm{H}_{2}$ at $E=0.93 \mathrm{eV}$ as a function of $\phi_{12}$ and $s$. The initial state is a superposition of $v=0, j=2, m_{1}=1, m_{2}=0$.

Faraday Discuss., 1999, 113, 291-302 


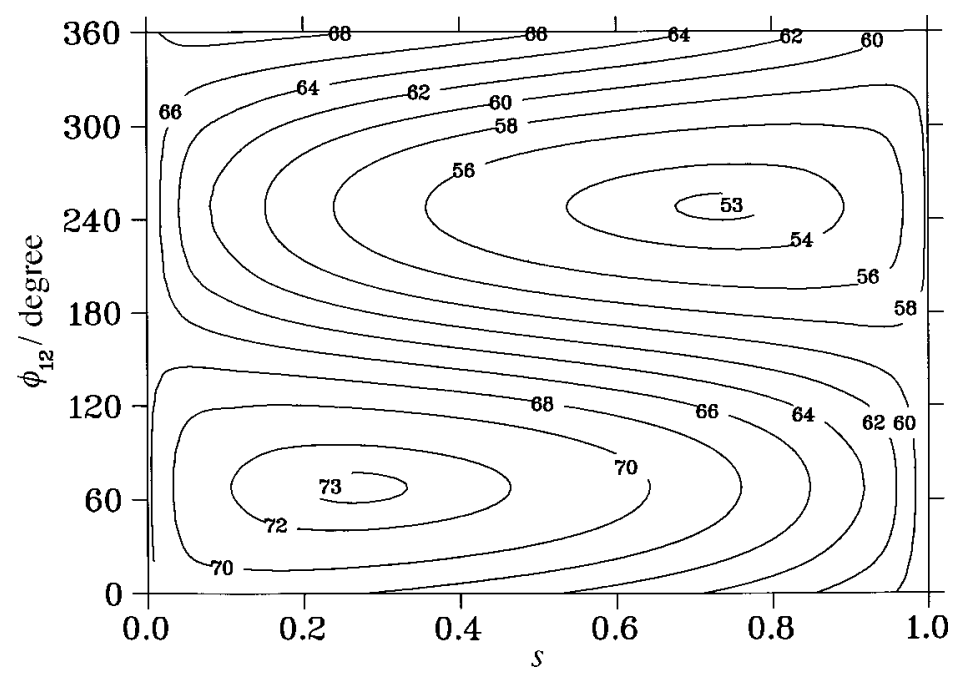

Fig. 3 Contour plot of the ratio $\sigma^{\mathrm{R}}(\varphi \leqslant \pi) / \sigma^{\mathrm{NR}}(\varphi \leqslant \pi)\left(\times 10^{3}\right)$ for $\mathrm{D}+\mathrm{H}_{2}$ at $E=1.25 \mathrm{eV}$ as a function of $\phi_{12}$ and $s$. The initial state is a superposition of $v=1, j=2, m_{1}=1, m_{2}=0$.

for scattering from a single $m_{i}$ state. Thus, the ratio of cross sections can be increased or decreased by $\approx 20 \%$ through coherent control effects. Somewhat better control is observed at higher energies $(E=1.25 \mathrm{eV})$, further from the reaction threshold (Fig. 3) where a superposition of $(v=1$, $j=2, m_{1}=1, m_{2}=0$ ) initial superposition states shows the ratio of cross sections varying from 0.053 to 0.073 as both $\phi_{12}$ and $s$ are varied.

In essence, superposing two $m$ levels provides some degree of control over the differential cross sections. Nonetheless, control is far from extensive. The origin of this behavior is evident from the sample results shown in Table 1, which shows the contributions $\sigma_{i i}$ and $\sigma_{12}$ for reactive and nonreactive scattering from several initial superposition states at $E=1.25 \mathrm{eV}$. The interference contributions $\sigma_{12}$ are seen to be, for both the reactive and nonreactive terms, approximately one order of magnitude smaller than the diagonal terms. As a consequence, the extent of control is rather limited. Two alternatives for improved control suggest themselves. The first is to seek alternate linear superpositions, e.g. composed from nondegenerate molecular states, ${ }^{9}$ whose resultant interference terms are closer in magnitude to the diagonal elements. The second is to examine the extent of control resulting from the inclusion of more than two degenerate reactant states in the initial superposition, as discussed below.

\section{B. Optimized bimolecular scattering}

The treatment above can be readily extended to superpositions composed of more than two states. In particular, we adopt a previously devised scheme to optimize the reactive cross section as a function of $c_{m}$ for an arbitrary number of states. ${ }^{22}$

Consider scattering from incident state $|E, q, \boldsymbol{n} ; 0\rangle$ to final state $\left|E, q^{\prime}, \boldsymbol{m} ; 0\right\rangle$. To simplify the notation we identify the $\boldsymbol{m}$ and $\boldsymbol{n}$ labels with a single quantum number, denoted $i$ and $f$, with

Table 1 Integral cross sections $\sigma_{i j}^{\mathrm{NR}, \mathrm{R}}(\varphi \leqslant \pi), i \leqslant j=1,2$, for $\mathrm{D}+\mathrm{H}_{2}$ at $E=1.25 \mathrm{eV}^{a}$

\begin{tabular}{llllllllll}
\hline$v$ & $j$ & $m_{1}$ & $m_{2}$ & $\sigma_{11}^{\mathrm{NR}}$ & $\sigma_{22}^{\mathrm{NR}}$ & $\sigma_{12}^{\mathrm{NR}}$ & $\sigma_{11}^{\mathrm{R}}$ & $\sigma_{22}^{\mathrm{R}}$ & $\sigma_{12}^{\mathrm{R}}$ \\
\hline 0 & 2 & 1 & 0 & $2.312(+1)$ & $2.305(+1)$ & $4.294(-1)$ & $3.337(+0)$ & $3.364(+1)$ & $3.578(-1)$ \\
0 & 2 & 2 & 1 & $2.466(+1)$ & $2.312(+1)$ & $8.854(-1)$ & $2.051(+0)$ & $3.337(+0)$ & $8.347(-1)$ \\
1 & 2 & 1 & 0 & $6.051(+1)$ & $6.083(+1)$ & $2.265(-1)$ & $4.092(+0)$ & $3.549(+0)$ & $3.152(-1)$ \\
1 & 2 & 2 & 1 & $6.295(+1)$ & $6.051(+1)$ & $1.014(+0)$ & $1.841(+0)$ & $4.092(+0)$ & $1.077(+0)$
\end{tabular}

${ }^{a}$ Numbers in parentheses denote the power of ten by which the tabulated value should be multiplied. 
associated free states $|E, q, i ; 0\rangle$ and $\left|E, q^{\prime}, f ; 0\right\rangle$. The result is, however, completely general. In accord with eqn. (2), the probability $P\left(f, q^{\prime} ; i, q\right)$ of producing product in final state $\left|f, E, q^{\prime}\right\rangle$ having started in the initial state $|i, E, q\rangle$ is

$$
P\left(f, q^{\prime} ; i, q\right)=\left|S_{f i}\right|^{2}
$$

where $S_{f i}=\left\langle E, f, q^{\prime}|S| E, i, q\right\rangle$ and where $S$ is the scattering matrix for the process. The total probability $P\left(q^{\prime} ; i, q\right)$ of scattering into arrangement channel $q^{\prime}$, assuming $m$ open product states, is given by

$$
P\left(q^{\prime} ; i, q\right)=\sum_{f=1}^{m}\left|S_{f i}\right|^{2}
$$

To simplify the notation we have not carried an $E$ label in the probabilities: fixed energy $E$ is understood.

If we now consider scattering from an initial state $\left|E, q,\left\{c_{i}\right\}\right\rangle$ composed of a linear superposition of $k$ states [i.e., eqn. (5) with $N=k$ ] then the probability of forming $\left|E, q^{\prime}, f\right\rangle$ from this initial state is

$$
P\left(f, q^{\prime} ; c, q\right)=\left|\sum_{i=1} c_{i} S_{f i}\right|^{2} .
$$

and the total reactive scattering probability into channel $q^{\prime}, P\left(q^{\prime} ; \boldsymbol{c}, q\right)$ is

$$
P\left(q^{\prime} ; \boldsymbol{c}, q\right)=\sum_{f=1}^{m}\left|\sum_{i=1}^{k} c_{i} S_{f i}\right|^{2} .
$$

To simplify the notation we introduce the matrix $\boldsymbol{A}=\boldsymbol{S}_{q^{\prime}}^{\dagger} \boldsymbol{S}_{q^{\prime}}$ with elements $A_{i j}=\sum_{f=1}^{m} S_{f j}^{*} S_{f i}$ which allows us to rewrite eqn. (34) as

$$
P\left(q^{\prime} ; \boldsymbol{c}, q\right)=\boldsymbol{c}^{\dagger} \boldsymbol{A} \boldsymbol{c} .
$$

Here $\dagger$ denotes the Hermitian conjugate and the $q^{\prime}$ subscript on the $\boldsymbol{S}$ indicates that we are dealing with the submatrix of the $\boldsymbol{S}$ matrix associated with scattering into product channel $q^{\prime}$.

One can optimize scattering into arrangement channel $q^{\prime}$, with the normalization constraint $\sum_{i=1}^{k}\left|c_{i}\right|^{2}=1$, by requiring

$$
\frac{\partial}{\partial c_{k}^{*}}\left[P\left(q^{\prime} ; \boldsymbol{c}, q\right)-\lambda \sum_{i=1}^{k}\left|c_{i}\right|^{2}\right]=\frac{\partial}{\partial c_{k}^{*}}\left[\boldsymbol{c}^{\dagger} \boldsymbol{A} \boldsymbol{c}-\lambda \boldsymbol{c}^{\dagger} \boldsymbol{c}\right]=0
$$

where $\lambda$ is a Lagrange multiplier. Explicitly taking the derivative gives the result that the optimized coefficients $c_{\lambda}$ satisfy the eigenvalue equation

$$
A c_{\lambda}=\lambda c_{\lambda} .
$$

Additional labels may be necessary to account for degeneracies of the eigenvectors $\boldsymbol{c}_{\lambda}$. Hence, optimization is equivalent to solving eqn. (37).

We have applied this approach to obtain optimal $\boldsymbol{c}_{\lambda}$ for all $\mathrm{H}+\mathrm{H}_{2}$ isotopic variants. Typical results are those shown here for $\mathrm{H}+\mathrm{H}^{\prime} \mathrm{D}$ at $E=1.25 \mathrm{eV}$, where scattering is optimized in each of the two product channels. In each case, we considered optimization of both $\sigma^{\mathrm{NR}}$ and of $\sigma^{\mathrm{R}}$ as a means of maximizing (or minimizing) $\sigma^{\mathrm{R}} / \sigma^{\mathrm{NR}}$. In all cases, optimization of $\sigma^{\mathrm{R}}$ was found to provide superior results.

Results for the maximum of $\sigma^{\mathrm{R}} / \sigma^{\mathrm{NR}}$ into both product arrangements are shown in columns 5 of Table 2 for a set of even $j$, along with the corresponding nonreactive and reactive cross sections. Results were similar for odd values of $j$. Also shown, for comparison purposes, are the maximum uncontrolled ratio, denoted $\sigma_{u}^{\mathrm{R}} / \sigma_{\mathrm{u}}^{\mathrm{NR}}$, along with $\sigma_{\mathrm{u}}^{\mathrm{R}}$ and $\sigma_{\mathrm{u}}^{\mathrm{NR}}$. Optimal control results for the same collision processes, but where we minimize $\sigma^{\mathrm{R}}$, are presented in Table 3. Examination of Tables 2 and 3 shows a large range of possible control. For example, scattering into $q=2$ from $j=2$ ranges over a factor of two, from a maximum of $2.63 \times 10^{-2}$ to a minimum of $1.15 \times 10^{-2}$. A much greater range of control $\left(1.98 \times 10^{-2}\right.$ to $\left.1.51 \times 10^{-4}\right)$ is evident for scattering into $q=3$ with initial $j=10$. Indeed the range of control increases with $j$, a manifestation of the increasing 
Table 2 Controlled, $\sigma^{\mathrm{R}, \mathrm{NR}}(\varphi \leqslant \pi)$, and uncontrolled, $\sigma_{\mathrm{u}}^{\mathrm{R}, \mathrm{NR}}(\varphi \leqslant \pi)$, integral cross sections for the $\mathrm{H}+\mathrm{H}^{\prime} \mathrm{D}$ collisions at $E=1.25 \mathrm{eV}^{a}$ (results of $\sigma^{\mathrm{R}}$ maximization)

\begin{tabular}{rrllllll}
\hline$q$ & $j$ & $\sigma^{\mathrm{R}}$ & $\sigma^{\mathrm{NR}}$ & $\sigma^{\mathrm{R}} / \sigma^{\mathrm{NR}}$ & $\sigma_{\mathrm{u}}^{\mathrm{R}}$ & $\sigma_{\mathrm{u}}^{\mathrm{NR}}$ & $\sigma_{\mathrm{u}}^{\mathrm{R}} / \sigma_{\mathrm{u}}^{\mathrm{NR}}$ \\
\hline 2 & 2 & $1.70(+0)$ & $6.49(+1)$ & $2.63(-2)$ & $1.41(+0)$ & $6.25(+1)$ & $2.26(-2)$ \\
2 & 4 & $1.96(+0)$ & $6.92(+1)$ & $2.83(-2)$ & $1.39(+0)$ & $6.32(+1)$ & $2.20(-2)$ \\
2 & 6 & $2.10(+0)$ & $7.53(+1)$ & $2.79(-2)$ & $1.30(+0)$ & $6.47(+1)$ & $2.01(-2)$ \\
2 & 8 & $1.89(+0)$ & $8.37(+1)$ & $2.26(-2)$ & $1.02(+0)$ & $6.71(+1)$ & $1.52(-2)$ \\
2 & 10 & $6.08(-1)$ & $9.67(+1)$ & $6.29(-3)$ & $3.12(-1)$ & $7.12(+1)$ & $4.39(-3)$ \\
3 & 2 & $1.35(+0)$ & $6.39(+1)$ & $2.11(-2)$ & $1.10(+0)$ & $6.25(+1)$ & $1.76(-2)$ \\
3 & 4 & $1.58(+0)$ & $6.78(+1)$ & $2.34(-2)$ & $1.19(+0)$ & $6.32(+1)$ & $1.88(-2)$ \\
3 & 6 & $1.86(+0)$ & $7.37(+1)$ & $2.52(-2)$ & $1.28(+0)$ & $6.47(+1)$ & $1.98(-2)$ \\
3 & 8 & $2.06(+0)$ & $8.25(+1)$ & $2.50(-2)$ & $1.29(+0)$ & $6.71(+1)$ & $1.93(-2)$ \\
3 & 10 & $1.92(+0)$ & $9.70(+1)$ & $1.98(-2)$ & $1.06(+0)$ & $7.12(+1)$ & $1.49(-2)$
\end{tabular}

${ }^{a}$ In all cases the initial $v=0$. For each case shown, coefficients $c_{i}$ were obtained to maximize $\sigma^{\mathrm{R}}$. The $q=2$ arrangement corresponds to $\mathrm{H}^{\prime}+\mathrm{HD}$ and $q=3$ corresponds to $\mathrm{D}+\mathrm{HH}^{\prime}$. Numbers in parentheses denote the power of ten by which the tabulated value should be multiplied.

ability so suppress reactive scattering as the number of initial states increases. ${ }^{22}$ We were unable, however, to significantly suppress nonreactive scattering as a means of enhancing the maximal $\sigma^{\mathrm{R}} / \sigma^{\mathrm{NR}}$ ratio. Nonetheless, in some instances (e.g. $\left.q=2, j=10\right)$, the optimized $\sigma^{\mathrm{R}}$ was close to the theoretical maximum of twice the unoptimized $\sigma_{\mathrm{u}}^{\mathrm{R}}$.

Optimal control coefficients $c_{i}$ resulting from the optimization procedure are shown in Table 4 for a number of the cases in Table 2. The case of $j=1$ has been added to allow the simple verification of the argument below.

Of considerable interest is the question of whether the optimized $c_{i}$ coefficients merely define a new vector $|j, m ; R\rangle$ which is simply a ket vector in a rotated coordinate system. If so this would indicate that the optimum solution corresponds to a simple classical reorientation of the diatomic angular momentum vector. Examination of the results indicate that this is not the case. Specifically, a vector $|j, m ; R\rangle$ rotated by Euler angles $\alpha, \beta, \gamma$ is given by ${ }^{23}$

$$
|j, m ; R\rangle=\sum_{m^{\prime}}\left\langle j, m^{\prime} \mid j, m ; R\right\rangle\left|j, m^{\prime}\right\rangle=\sum_{m^{\prime}} D_{m^{\prime} m}^{(j)}(\alpha, \beta, \gamma)\left|j, m^{\prime}\right\rangle .
$$

Thus, if the result of the optimal control procedure were merely to affect a rotation of the $j$ vector, then the coefficients $c_{i}$ should equal (to within an overall phase) $D_{m^{\prime} m}^{(j)}(\alpha, \beta, \gamma)$. A careful exami-

Table 3 Controlled, $\sigma^{\mathrm{R}, \mathrm{NR}}(\varphi \leqslant \pi)$, and uncontrolled, $\sigma_{\mathrm{u}}^{\mathrm{R}, \mathrm{NR}}(\varphi \leqslant \pi)$, integral cross sections for the $\mathrm{H}+\mathrm{H}^{\prime} \mathrm{D}$ collisions at $E=1.25 \mathrm{eV}^{a}$ (results of $\sigma^{\mathrm{R}}$ minimization)

\begin{tabular}{rrllllll}
\hline$q$ & \multicolumn{1}{l}{$j$} & $\sigma^{\mathrm{R}}$ & $\sigma^{\mathrm{NR}}$ & $\sigma^{\mathrm{R}} / \sigma^{\mathrm{NR}}$ & $\sigma_{\mathrm{u}}^{\mathrm{R}}$ & $\sigma_{\mathrm{u}}^{\mathrm{NR}}$ & $\sigma_{\mathrm{u}}^{\mathrm{R}} / \sigma_{\mathrm{u}}^{\mathrm{NR}}$ \\
\hline 2 & 2 & $7.23(-1)$ & $6.26(+1)$ & $1.15(-2)$ & $8.62(-1)$ & $6.33(+1)$ & $1.36(-2)$ \\
2 & 4 & $4.70(-1)$ & $6.28(+1)$ & $7.49(-3)$ & $6.64(-1)$ & $6.45(+1)$ & $1.03(-2)$ \\
2 & 6 & $2.21(-1)$ & $6.31(+1)$ & $3.50(-3)$ & $4.07(-1)$ & $6.62(+1)$ & $6.15(-3)$ \\
2 & 8 & $3.59(-2)$ & $6.36(+1)$ & $5.65(-4)$ & $1.07(-1)$ & $6.85(+1)$ & $1.56(-3)$ \\
2 & 10 & $1.18(-3)$ & $6.40(+1)$ & $1.85(-5)$ & $5.60(-3)$ & $7.16(+1)$ & $7.82(-5)$ \\
3 & 2 & $3.58(-1)$ & $6.34(+1)$ & $5.65(-3)$ & $5.35(-1)$ & $6.33(+1)$ & $8.45(-3)$ \\
3 & 4 & $2.22(-1)$ & $6.41(+1)$ & $3.46(-3)$ & $4.06(-1)$ & $6.45(+1)$ & $6.29(-3)$ \\
3 & 6 & $1.22(-1)$ & $6.52(+1)$ & $1.87(-3)$ & $2.87(-1)$ & $6.62(+1)$ & $4.34(-3)$ \\
3 & 8 & $4.89(-2)$ & $6.67(+1)$ & $7.22(-4)$ & $1.51(-1)$ & $6.85(+1)$ & $2.20(-3)$ \\
3 & 10 & $1.03(-2)$ & $6.84(+1)$ & $1.51(-4)$ & $4.27(-2)$ & $7.16(+1)$ & $5.96(-4)$
\end{tabular}

${ }^{a}$ In all cases the initial $v=0$. For each case shown, coefficients $c_{i}$ were obtained to minimize $\sigma^{\mathrm{R}}$. The $q=2$ arrangement corresponds to $\mathrm{H}^{\prime}+\mathrm{HD}$ and $q=3$ corresponds to $\mathrm{D}+\mathrm{HH}^{\prime}$. Numbers in parentheses denote the power of ten by which the tabulated value should be multiplied. 
Table 4 Optimal control coefficients $c_{i}$, for the $\mathrm{H}+\mathrm{H}^{\prime} \mathrm{D}$ collisions at $E=1.25 \mathrm{eV}^{a}$

\begin{tabular}{|c|c|c|c|c|c|c|c|c|c|}
\hline \multirow[b]{2}{*}{$q$} & \multirow[b]{2}{*}{$m_{i}$} & \multicolumn{2}{|c|}{$v=0, j=1$} & \multicolumn{2}{|c|}{$v=0, j=2$} & \multicolumn{2}{|c|}{$v=0, j=4$} & \multicolumn{2}{|c|}{$v=0, j=6$} \\
\hline & & $\left|c_{i}\right|$ & $\arg \left(c_{i}\right)$ & $\left|c_{i}\right|$ & $\arg \left(c_{i}\right)$ & $\left|c_{i}\right|$ & $\arg \left(c_{i}\right)$ & $\left|c_{i}\right|$ & $\arg \left(c_{i}\right)$ \\
\hline 2 & 0 & 0.914 & 0.429 & 0.592 & 1.958 & 0.576 & -2.250 & 0.542 & -0.756 \\
\hline 2 & 1 & 0.287 & $0.0[+0]$ & 0.540 & $0.476[-\pi]$ & 0.494 & $2.539[-\pi]$ & 0.478 & $-2.273[+\pi]$ \\
\hline 2 & 2 & & & 0.183 & $0.0[+0]$ & 0.278 & $1.341[-\pi]$ & 0.312 & $2.624[+0]$ \\
\hline 2 & 3 & & & & & 0.112 & $0.381[-\pi]$ & 0.155 & $1.393[-\pi]$ \\
\hline 2 & 4 & & & & & 0.028 & $0.0[+0]$ & 0.052 & $0.385[+0]$ \\
\hline 2 & 5 & & & & & & & 0.014 & $-0.212[+\pi]$ \\
\hline 2 & 6 & & & & & & & 0.003 & $0.0[+0]$ \\
\hline 3 & 0 & 0.890 & -2.973 & 0.535 & 0.984 & 0.498 & -0.834 & 0.500 & 0.117 \\
\hline 3 & 1 & 0.322 & $-\pi\lceil+\pi\rceil$ & 0.561 & $0.194[-\pi]$ & 0.468 & $-2.061[+\pi]$ & 0.453 & $-1.256\lceil+\pi]$ \\
\hline 3 & 2 & & & 0.206 & $0.0[+0]$ & 0.333 & $-2.775[+0]$ & 0.328 & $-2.232[+0]$ \\
\hline 3 & 3 & & & & & 0.205 & $-3.104[+\pi]$ & 0.213 & $-2.877[+\pi]$ \\
\hline 3 & 4 & & & & & 0.063 & $-\pi[+0]$ & 0.118 & $3.005[+0]$ \\
\hline 3 & 5 & & & & & & & 0.052 & $2.882[-\pi]$ \\
\hline 3 & 6 & & & & & & & 0.012 & $-\pi[+0]$ \\
\hline
\end{tabular}

${ }^{a} q=2$ corresponds to the final $\mathrm{H}^{\prime}+\mathrm{HD}$ arrangement and $q=3$ corresponds to the final $\mathrm{D}+\mathrm{HH}^{\prime}$ arrangement. Here $c_{i}=c_{m}$ where $-j \leqslant m_{i} \leqslant j$. Values of $\left|c_{i}\right|$ are found to be a function of $\left|m_{i}\right|$ and $\arg \left(c_{i}\right)$ for $\left|m_{i}\right|$ are shown. In order to get $\arg \left(c_{i}\right)$ for $-\left|m_{i}\right|$, one should add the numbers in square brackets to the value of $\arg \left(c_{i}\right)$ for $\left|m_{i}\right| .\left[\right.$ e.g. $\arg \left(c_{i}\right)=0$ for $\left.q=3, m_{i}=-1, v=0, j=1\right]$.

nation of the results showed that this is not the case. Thus, optimal control results from quantum interference effects and not from a simple classical reorientation of the angular momentum vector.

\section{Summary}

Our general treatment of laboratory conditions for bimolecular control has identified two distinctly different classes of control. In the first, one prepares a superposition of degenerate internal reactant states which is subsequently used as a reagent in the collision. In this case there are no undesirable uncontrolled satellite contributions. However, in examining the most natural case, i.e. superposing diatomic $m$ states in collisions with atoms, we have found that (a) one can control differential, but not total integral, cross sections and (b) control, although evident in general, only becomes extensive with the inclusion of many initial $m$ states. An examination of Table 1 shows that this is because the $\sigma_{12}$ interference contributions are small when compared to the $\sigma_{i i}$.

The second control class requires the creation of the desired superposition state [eqn. (5)] from non-degenerate internal states of the reactants. In this instance we require special conditions in the laboratory in which the relative motion of the atom and internal energy are correlated within a laboratory superposition state. Although similar correlations have been established in laser cooling of atoms ${ }^{11}$ these techniques have yet to be extended to molecules. Given these conditions controllable superpositions result, but they are accompanied by uncontrolled satellite terms [e.g. eqn. (16)]. Nonetheless, previous studies on model collinear scattering ${ }^{9}$ suggest the possibility of extensive control under these circumstances due to large relative values of $\sigma_{12}$. These studies motivate the further work which is currently underway ${ }^{24}$ on three-dimensional atom-diatom scattering using non-degenerate internal states in the initial superposition state.

\section{Acknowledgements}

We thank Professors R. Wyatt and B. Ramachandran for providing us with the REACT scattering code and for useful comments on its use. This work was supported in part by the U.S. Office of Naval Research, Photonics Research Ontario and by a grant of HPC time on the CRAY C-90 and the SGI Origin 2000 from the DoD ASC MSRC Center, and the CRAY T-90 and the SGI PCA from the DoD NAVOCEANO MSRC Center. 


\section{References}

1 M. Shapiro and P. Brumer, J. Chem. Soc., Faraday Trans., 1997, 93, 1263; P. Brumer and M. Shapiro, Annu. Rev. Phys. Chem., 1992, 43, 257.

2 B. Kohler, J. L. Krause, F. Raski, K. R. Wilson, V. V. Yakovler, R. M. Whitnell and Y. Yan, Acc. Chem. Res., 1995, 28, 133; W. S. Warren, H. Rabitz and M. Dahleh, Science, 1993, 259, 1581; S. A. Rice, ibid., $1992,258,412$.

3 See, e.g., L. Zhu, V. Kleiman, X. Li, S-P. Liu, K. Trentelman and R. J. Gordon, Science, 1995, 270, 77, and references therein.

4 E. Dupont, P. B. Corkum, H. C. Liu, M. Buchanan and Z. R. Wasilewski, Phys. Rev. Lett., 1995, 74, 3596.

5 A. Shnitman, I. Sofer, I. Golub, A. Yogev, M. Shapiro, Z. Chen and P. Brumer, Phys. Rev. Lett., 1996, 76, 2886.

6 M. Shapiro and P. Brumer, Phys. Rev. Lett., 1996, 77, 2574.

7 A. Abrashkevich, M. Shapiro and P. Brumer, Phys. Rev. Lett., 1998, 81, 3789.

8 An erratum (A. Abrashkevich, M. Shapiro and P. Brumer, Phys. Rev. Lett., 1999, 82, 3002) clarifies that the results shown in ref. 7 are valid at $\phi=0$ and that control over the total cross section is not possible when building the initial superposition from helicity states.

9 D. Holmes, M. Shapiro and P. Brumer, J. Chem. Phys., 1996, 105, 9162.

10 Atom Interferometry, ed. P. Berman, Academic Press, San Diego, 1997.

11 J. Lawall, F. Bardou, K. Shimizu, M. Ledue, A. Aspect and C. Cohen-Tannoudji, Phys. Rev. Lett., 1994, 73, 1915.

12 J. R. Taylor, Scattering Theory, Wiley, New York, 1972.

13 This is not to say that the oscillatory interference term cannot be put to good use. See, for example, our proposal for generating THz radiation: P. Brumer and M. Shapiro, in Coherent Control in Atoms, Molecules and Semiconductors, ed. W. Pötz and W. A. Schroeder, Kluwer, Dordrecht, 1999.

14 G. C. Schatz and A. Kuppermann, J. Chem. Phys., 1976, 65, 4642; eqn. (21) of our paper corrects a typographical error in the seminal Schatz-Kuppermann paper (specifically, $m_{i}$ should appear in the exponent and not $k^{\prime}$ ).

15 R. T. Pack and G. A. Parker, J. Chem. Phys., 1987, 87, 3888.

16 J. Z. H. Zhang and W. H. Miller, J. Chem. Phys., 1989, 91, 1528.

17 W. H. Miller, J. Chem. Phys., 1969, 50, 407.

18 J. L. Krause, M. Shapiro and P. Brumer, J. Chem. Phys., 1990, 92, 1126

19 P. Siegbahn and B. Liu, J. Chem. Phys., 1978, 68, 2457; D. G. Truhlar and C. J. Horowitz, ibid., 1978, 68, 2466; 1979, 71, 1514.

20 D. E. Manolopoulos and R. E. Wyatt, Chem. Phys. Lett., 1988, 152, 23.

21 D. E. Manolopoulos, M. D'Mello and R. E. Wyatt, J. Chem. Phys., 1990, 93, 403.

22 E. Frishman, M. Shapiro and P. Brumer, J. Chem. Phys., 1999, 110, 9.

23 A. R. Edmonds, Angular Momentum in Quantum Mechanics, Princeton University Press, Princeton, 1960.

24 A. Abrashkevich, M. Shapiro and P. Brumer, work in progress.

Paper 9/02135C 